\title{
High-resolution microwave studies of ring-structured complexes between trifluoroacetic acid and water
}

\author{
Bin Ouyang, Tony G. Starkey, and Brian J. Howard* \\ Physical and Theoretical Chemistry Laboratory, South Parks Road, University of Oxford, \\ Oxford OX1 3QZ, United Kingdom
}

\section{Supplementary Material}

\section{A. Observed transition lines}

Tables S.1 - S.4 list the observed transition lines of TFA, TFA $-\mathrm{H}_{2} \mathrm{O}$, TFA- $\left(\mathrm{H}_{2} \mathrm{O}\right)_{2}$ and TFA- $\left(\mathrm{H}_{2} \mathrm{O}\right)_{3}$, respectively.

Note that for the TFA- $\mathrm{H}_{2} \mathrm{O}$, some of the lines have been splitted due to the presence of the tunneling motions. The transition frequencies listed in Table S.1 are those of the state with nuclear spin statistics $I=3$. The frequencies of the other state with nuclear spin statistics $I=1$ can be easily acquired by subtracting the splittings listed in Table 4 from those of the state with $I=3$.

\section{B. Vibrational Frequencies}

The vibrational frequencies of the TFA monomers have been well documented in the literature [1] except for the lowest one which corresponds to the torsion of the $\mathrm{CF}_{3}-$ group. We averaged the frequencies from the different papers to get the experimental values for the purpose of scaling their theoretical counterparts. It should be noted that if the frequency reported in one paper is in serious disagreement with others, we have chosen to exclude it from the averaging calculations. The theoretical vibrational frequencies of the TFA monomer were calculated on HF/6-31G(d) level, and were thereafter scaled according to two schemes, one to minimize the errors in the low-frequency region (for partition function calculations) and the other to minimize the errors in the high-frequency region (for zero point vibrational energy calculations) [2]. The experimental and the scaled theoretical vibrational frequencies of the TFA monomer are tabulated in Table S.5. Those of the $\mathrm{H}_{2} \mathrm{O}$ are listed in the same table as well. The experimental vibrational frequencies of the $\mathrm{H}_{2} \mathrm{O}$ monomer were taken from reference [3]. Although the HF/6-31G(d) method applied looks rather modest, the performance of it in predicting the vibrational frequencies of the two monomers is fairly satisfactory, as shown in Table S.5.

This method was further extended to predict the vibrational frequencies of the global minimal conformation of the three complexes. The monomer vibrational frequencies retained in the complexes were scaled by their respective scaling factors, while all the inter-monomer frequencies were scaled by that of

\footnotetext{
*Author to whom correspondence should be addressed. FAX: +44 1865 275410. E-mail: brian.howard@chem.ox.ac.uk
} 
the TFA monomer. The results have been summarized in Table S.6.

\section{References}

[1] (a) Fuson, N.; Josien, M.-L.; Jones, E. A.; Lawson, J. R. J. Chem. Phys. 1952, 20, 1627. (b) Kagarise, R. E.; J. Chem. Phys. 1957, 27, 519. (c) Barceló, J. R.; Otero, C.Spectrochim. Acta 1962, $18,1231$. (d) Redington, R. L.; Lin, K. C. Spectrochim. Acta 1971, 27 A, 2445.

[2] Scott, A. P.; Radom, L. J. Phys. Chem. A 1996, 100, 16502.

[3] Herzberg, G. Infrared and Raman Spectra of Polyatomic Molecules; D. Van Nostrand Company, Inc.: New York, 1945. 
Table S.1: Observed transition lines of the TFA monomer.

\begin{tabular}{crr}
\hline \hline Transition & $\nu_{\text {obs }} / \mathrm{MHz}$ & $\Delta \nu^{a} / \mathrm{MHz}$ \\
\hline$a-$ type & & \\
$2_{0,2} \leftarrow 1_{0,1}$ & $9,063.8111$ & -0.0001 \\
$2_{1,1} \leftarrow 1_{1,0}$ & $9,571.5572$ & 0.0005 \\
$2_{1,2} \leftarrow 1_{1,1}$ & $8,724.3744$ & -0.0004 \\
$3_{0,3} \leftarrow 2_{0,2}$ & $13,405.1381$ & -0.0008 \\
$3_{1,2} \leftarrow 2_{1,1}$ & $14,298.3160$ & 0.0001 \\
$3_{1,3} \leftarrow 2_{1,2}$ & $13,038.2572$ & 0.0004 \\
$3_{2,1} \leftarrow 2_{2,0}$ & $14,038.6874$ & 0.0004 \\
$4_{0,4} \leftarrow 3_{0,3}$ & $17,599.8695$ & 0.0000 \\
$4_{1,4} \leftarrow 3_{1,3}$ & $17,307.5966$ & 0.0005 \\
$3_{2,2} \leftarrow 3_{0,3}$ & $6,713.4389$ & 0.0018 \\
$b-$ type & & \\
$2_{1,2} \leftarrow 1_{0,1}$ & $10,090.7163$ & 0.0001 \\
$2_{0,2} \leftarrow 1_{1,1}$ & $7,697.4681$ & -0.0017 \\
$2_{2,1} \leftarrow 1_{1,0}$ & $13,670.5812$ & 0.0018 \\
$2_{2,0} \leftarrow 1_{1,1}$ & $14,178.3369$ & -0.0021 \\
$3_{1,3} \leftarrow 2_{0,2}$ & $14,065.1625$ & 0.0005 \\
$3_{0,3} \leftarrow 2_{1,2}$ & $12,378.2340$ & -0.0004 \\
$3_{2,2} \leftarrow 2_{1,1}$ & $17,820.8958$ & -0.0012 \\
$3_{1,2} \leftarrow 2_{2,1}$ & $10,199.2925$ & -0.0006 \\
$4_{1,4} \leftarrow 3_{0,3}$ & $17,967.6185$ & -0.0009 \\
$4_{0,4} \leftarrow 3_{1,3}$ & $16,939.8469$ & 0.0008 \\
$3_{3,0} \leftarrow 3_{2,1}$ & $7,548.7254$ & -0.0007 \\
$3_{3,1} \leftarrow 3_{2,2}$ & $7,938.9996$ & 0.0017 \\
$4_{1,3} \leftarrow 3_{2,2}$ & $15,416.8597$ & -0.0006 \\
$4_{2,3} \leftarrow 4_{1.4}$ & $6,975.4114$ & -0.0005 \\
$4_{3,2} \leftarrow 4_{2,3}$ & $8,146.1869$ & -0.0001 \\
$4_{4,0} \leftarrow 4_{3,1}$ & $10,851.7823$ & -0.0016 \\
$4_{4,1} \leftarrow 4_{3,2}$ & $10,923.8602$ & -0.0060 \\
$5_{2,4} \leftarrow 5_{1,5}$ & $8,123.2184$ & -0.0001 \\
$5_{3,3} \leftarrow 5_{2,4}$ & $8,527.8182$ & -0.0014 \\
$5_{4,2} \leftarrow 5_{3,3}$ & $10,915.2151$ & 0.0023 \\
$5_{4,1} \leftarrow 5_{3,2}$ & $10,646.1814$ & 0.0012 \\
$6_{2,5} \leftarrow 6_{1,6}$ & $9,467.0594$ & -0.0030 \\
$7_{1,6} \leftarrow 7_{0,7}$ & $10,215.1522$ & 0.0005 \\
\hline \hline$a$ & &
\end{tabular}

${ }^{a} \Delta \nu=\nu_{\text {obs }}-\nu_{\text {calc }}$. 
Table S.2: Observed transition lines of the TFA- $\left(\mathrm{H}_{2} \mathrm{O}\right)$ complex. ${ }^{a}$

\begin{tabular}{|c|c|c|}
\hline Transition & $\nu_{\text {obs }} / \mathrm{MHz}$ & $\Delta \nu^{a} / \mathrm{MHz}$ \\
\hline$a-$ type & & \\
\hline $4_{0,4} \leftarrow 3_{0,3}$ & $8,284.3470$ & -0.0004 \\
\hline $4_{1,3} \leftarrow 3_{1,2}$ & $8,478.6772$ & 0.0013 \\
\hline $4_{1,4} \leftarrow 3_{1,3}$ & $8,123.6835$ & 0.0015 \\
\hline $4_{2,2} \leftarrow 3_{2,1}$ & $8,324.8160$ & -0.0005 \\
\hline $4_{2,3} \leftarrow 3_{2,2}$ & $8,303.7386$ & -0.0016 \\
\hline $5_{0,5} \leftarrow 4_{0,4}$ & $10,339.8148$ & -0.0004 \\
\hline $5_{1,4} \leftarrow 4_{1,3}$ & $10,594.1300$ & 0.0014 \\
\hline $5_{1,5} \leftarrow 4_{1,4}$ & $10,150.8183$ & 0.0001 \\
\hline $5_{2,3} \leftarrow 4_{2,2}$ & $10,418.9311$ & -0.0004 \\
\hline $5_{2,4} \leftarrow 4_{2,3}$ & $10,376.9958$ & -0.0005 \\
\hline $5_{3,2} \leftarrow 4_{3,1}$ & $10,389.2649$ & -0.0003 \\
\hline $5_{3,3} \leftarrow 4_{3,2}$ & $10,388.6054$ & 0.0014 \\
\hline $5_{4,2} \leftarrow 4_{4,1}$ & $10,386.3066$ & 0.0013 \\
\hline $6_{0,6} \leftarrow 5_{0,5}$ & $12,385.3372$ & -0.0007 \\
\hline $6_{1,5} \leftarrow 5_{1,4}$ & $12,706.5985$ & 0.0015 \\
\hline $6_{1,6} \leftarrow 5_{1,5}$ & $12,175.5957$ & 0.0004 \\
\hline $6_{2,4} \leftarrow 5_{2,3}$ & $12,521.1829$ & 0.0000 \\
\hline $6_{2,5} \leftarrow 5_{2,4}$ & $12,448.4680$ & -0.0013 \\
\hline $6_{3,3} \leftarrow 5_{3,2}$ & $12,470.4674$ & -0.0005 \\
\hline $6_{3,4} \leftarrow 5_{3,3}$ & $12,468.7068$ & -0.0005 \\
\hline $7_{0,7} \leftarrow 6_{0,6}$ & $14,419.6720$ & -0.0003 \\
\hline $7_{1,6} \leftarrow 6_{1,5}$ & $14,815.3023$ & 0.0006 \\
\hline $7_{1,7} \leftarrow 6_{1,6}$ & $14,197.7071$ & -0.0002 \\
\hline $7_{2,5} \leftarrow 6_{2,4}$ & $14,632.4455$ & 0.0005 \\
\hline $7_{2,6} \leftarrow 6_{2,5}$ & $14,517.8061$ & -0.0018 \\
\hline $7_{3,4} \leftarrow 6_{3,3}$ & $14,553.7895$ & -0.0022 \\
\hline $7_{3,5} \leftarrow 6_{3,4}$ & $14,549.8381$ & -0.0014 \\
\hline $7_{4,3} \leftarrow 6_{4,2}$ & $14,544.6786$ & 0.0015 \\
\hline $7_{4,4} \leftarrow 6_{4,3}$ & $14,544.6290$ & -0.0014 \\
\hline $8_{0,8} \leftarrow 7_{0,7}$ & $16,442.2393$ & -0.0003 \\
\hline $8_{1,8} \leftarrow 7_{1,7}$ & $16,216.9227$ & 0.0006 \\
\hline $8_{2,6} \leftarrow 7_{2,5}$ & $16,752.9200$ & -0.0005 \\
\hline $8_{2,7} \leftarrow 7_{2,6}$ & $16,584.6678$ & 0.0015 \\
\hline $8_{3,5} \leftarrow 7_{3,4}$ & $16,639.8346$ & 0.0003 \\
\hline $8_{3,6} \leftarrow 7_{3,5}$ & $16,631.9590$ & 0.0003 \\
\hline $8_{5,4} \leftarrow 7_{5,3}$ & $16,620.5646$ & 0.0009 \\
\hline
\end{tabular}

${ }^{a}$ Some of the transitions are splitted due to tunneling motions (section 3.3). We listed here only the transition frequencies for one of the states with $I=3$.

${ }^{b} \Delta \nu=\nu_{\text {obs }}-\nu_{\text {calc }}$. 
Table S.3: Observed transition lines of the TFA- $\left(\mathrm{H}_{2} \mathrm{O}\right)_{2}$ complex.

\begin{tabular}{crr}
\hline \hline Transition & $\nu_{\text {obs }} / \mathrm{MHz}$ & \multicolumn{1}{c}{$\Delta \nu^{a} / \mathrm{MHz}$} \\
\hline$a-$ type & & \\
$5_{0,5} \leftarrow 4_{0,4}$ & $6,632.2946$ & -0.0001 \\
$5_{1,4} \leftarrow 4_{1,3}$ & $6,927.4715$ & -0.0003 \\
$5_{2,3} \leftarrow 4_{2,2}$ & $6,768.9722$ & 0.0006 \\
$6_{0,6} \leftarrow 5_{0,5}$ & $7,921.7406$ & 0.0003 \\
$6_{1,6} \leftarrow 5_{1,5}$ & $7,730.6225$ & 0.0007 \\
$6_{2,4} \leftarrow 5_{2,3}$ & $8,152.9241$ & 0.0004 \\
$6_{2,5} \leftarrow 5_{2,4}$ & $8,029.0115$ & 0.0000 \\
$6_{3,3} \leftarrow 5_{3,2}$ & $8,068.5681$ & -0.0001 \\
$6_{3,4} \leftarrow 5_{3,3}$ & $8,063.5903$ & 0.0011 \\
$7_{0,7} \leftarrow 6_{0,6}$ & $9,195.1466$ & 0.0006 \\
$7_{1,6} \leftarrow 6_{1,5}$ & $9,668.0654$ & -0.0001 \\
$7_{1,7} \leftarrow 6_{1,6}$ & $9,007.5456$ & 0.0005 \\
$7_{2,5} \leftarrow 6_{2,4}$ & $9,549.1345$ & 0.0008 \\
$7_{2,6} \leftarrow 6_{2,5}$ & $9,357.8414$ & 0.0001 \\
$7_{3,4} \leftarrow 6_{3,3}$ & $9,423.0912$ & -0.0010 \\
$7_{3,5} \leftarrow 6_{3,4}$ & $9,411.9583$ & 0.0001 \\
$7_{4,3} \leftarrow 6_{4,2}$ & $9,405.0742$ & -0.0015 \\
$7_{4,4} \leftarrow 6_{4,3}$ & $9,404.8613$ & 0.0002 \\
$8_{0,8} \leftarrow 7_{0,7}$ & $10,280.2192$ & 0.0000 \\
$8_{1,7} \leftarrow 7_{1,6}$ & $11,025.7573$ & -0.0002 \\
$8_{1,8} \leftarrow 7_{1,7}$ & $10,280.2192$ & 0.0000 \\
$8_{2,6} \leftarrow 7_{2,5}$ & $10,955.3289$ & -0.0003 \\
$8_{2,7} \leftarrow 7_{2,6}$ & $10,682.4264$ & -0.0019 \\
$8_{3,5} \leftarrow 7_{3,4}$ & $10,783.3665$ & 0.0005 \\
$8_{3,6} \leftarrow 7_{3,5}$ & $10,761.3101$ & 0.0002 \\
$8_{4,4} \leftarrow 7_{4,3}$ & $10,753.7628$ & 0.0003 \\
$8_{4,5} \leftarrow 7_{4,4}$ & $10,753.1729$ & -0.0012 \\
$8_{5,3} \leftarrow 7_{5,2}$ & $10,745.4562$ & 0.0013 \\
$8_{5,4} \leftarrow 7_{5,3}$ & $10,745.4477$ & 0.0000 \\
$9_{0,9} \leftarrow 8_{0,8}$ & $11,701.7137$ & 0.0001 \\
$9_{1,8} \leftarrow 8_{1,7}$ & $12,372.3448$ & -0.0014 \\
$9_{1,9} \leftarrow 8_{1,8}$ & $11,548.6901$ & -0.0003 \\
$9_{2,7} \leftarrow 8_{2,6}$ & $12,367.6265$ & 0.0016 \\
$9_{2,8} \leftarrow 8_{2,7}$ & $12,002.2457$ & 0.0010 \\
\hline & &
\end{tabular}




\begin{tabular}{crr}
\hline Transition & $\nu_{\text {obs }} / \mathrm{MHz}$ & $\Delta \nu^{a} / \mathrm{MHz}$ \\
\hline$a-$ type & & \\
$9_{3,6} \leftarrow 8_{3,5}$ & $12,150.9970$ & 0.0002 \\
$9_{3,7} \leftarrow 8_{3,6}$ & $12,111.1299$ & -0.0014 \\
$9_{4,5} \leftarrow 8_{4,4}$ & $12,104.6696$ & 0.0012 \\
$9_{4,6} \leftarrow 8_{4,5}$ & $12,103.2614$ & -0.0006 \\
$10_{0,10} \leftarrow 9_{0,9}$ & $12,941.8502$ & 0.0000 \\
$10_{1,9} \leftarrow 9_{1,8}$ & $13,705.6221$ & -0.0011 \\
$10_{1,10} \leftarrow 9_{1,9}$ & $12,813.1710$ & 0.0049 \\
$10_{2,8} \leftarrow 9_{2,7}$ & $13,781.3490$ & -0.0003 \\
$10_{2,9} \leftarrow 9_{2,8}$ & $13,316.8209$ & -0.0002 \\
$10_{3,7} \leftarrow 9_{3,6}$ & $13,527.7123$ & -0.0007 \\
$10_{3,8} \leftarrow 9_{3,7}$ & $13,460.7510$ & 0.0013 \\
$11_{0,11} \leftarrow 10_{0,10}$ & $14,178.0817$ & 0.0014 \\
$11_{1,10} \leftarrow 10_{1,9}$ & $15,023.4657$ & 0.0010 \\
$11_{1,11} \leftarrow 10_{1,10}$ & $14,073.9696$ & -0.0097 \\
$11_{2,9} \leftarrow 10_{2,8}$ & $15,191.9753$ & 0.0003 \\
$11_{2,10} \leftarrow 10_{2,9}$ & $14,625.7604$ & -0.0004 \\
$11_{3,8} \leftarrow 10_{3,7}$ & $14,915.1477$ & 0.0011 \\
$11_{5,6} \leftarrow 10_{5,5}$ & $14,791.1131$ & -0.0005 \\
$11_{5,7} \leftarrow 10_{5,6}$ & $14,790.9516$ & -0.0007 \\
$12_{0,12} \leftarrow 11_{0,11}$ & $15,413.0981$ & -0.0001 \\
$12_{1,12} \leftarrow 11_{1,11}$ & $15,331.5451$ & -0.0021 \\
$12_{2,10} \leftarrow 11_{2,9}$ & $16,595.7087$ & 0.0006 \\
$12_{3,9} \leftarrow 11_{3,8}$ & $16,314.4738$ & -0.0015 \\
$13_{0,13} \leftarrow 12_{0,12}$ & $16,648.5662$ & 0.0011 \\
$13_{1,12} \leftarrow 12_{1,11}$ & $17,606.9851$ & -0.0005 \\
$13_{2,12} \leftarrow 12_{2,11}$ & $17,225.6710$ & -0.0015 \\
$13_{3,11} \leftarrow 12_{3,10}$ & $17,500.1010$ & 0.0015 \\
\hline \hline$a_{2}=\nu_{0}-\nu_{c}$ & &
\end{tabular}

${ }^{a} \Delta \nu=\nu_{\mathrm{obs}}-\nu_{\text {calc }}$. 
Table S.4: Observed transition lines of the TFA- $\left(\mathrm{H}_{2} \mathrm{O}\right)_{3}$ complex

\begin{tabular}{|c|c|c|}
\hline Transition & $\nu_{\text {obs }} / \mathrm{MHz}$ & $\Delta \nu^{a} / \mathrm{MHz}$ \\
\hline$a-$ type & & \\
\hline $7_{2,5} \leftarrow 6_{2,4}$ & 6510.3294 & -0.0083 \\
\hline $8_{1,8} \leftarrow 7_{1,7}$ & $6,890.1303$ & -0.0040 \\
\hline $8_{0,8} \leftarrow 7_{0,7}$ & $7,009.3861$ & -0.0134 \\
\hline $8_{2,7} \leftarrow 7_{2,6}$ & $7,220.9368$ & 0.0103 \\
\hline $8_{3,6} \leftarrow 7_{3,5}$ & $7,296.1534$ & -0.0091 \\
\hline $8_{3,5} \leftarrow 7_{3,4}$ & $7,321.612$ & -0.0903 \\
\hline $8_{2,6} \leftarrow 7_{2,5}$ & $7,475.8554$ & -0.0032 \\
\hline $8_{1,7} \leftarrow 7_{1,6}$ & $7,493.7345$ & -0.0063 \\
\hline $9_{1,9} \leftarrow 8_{1,8}$ & $7,736.2943$ & 0.0012 \\
\hline $9_{0,9} \leftarrow 8_{0,8}$ & $7,835.9773$ & -0.0135 \\
\hline $9_{2,8} \leftarrow 8_{2,7}$ & $8,108.4859$ & 0.0203 \\
\hline $9_{3,7} \leftarrow 8_{3,6}$ & $8,211.5716$ & -0.0107 \\
\hline $9_{4,5} \leftarrow 8_{4,4}$ & $8,208.6722$ & -0.0342 \\
\hline $9_{1,8} \leftarrow 8_{1,7}$ & $8,398.155$ & -0.0031 \\
\hline $9_{2,7} \leftarrow 8_{2,6}$ & $8,443.7611$ & -0.0037 \\
\hline $10_{1,10} \leftarrow 9_{1,9}$ & $8,579.1077$ & 0.0016 \\
\hline $10_{0,10} \leftarrow 9_{0,9}$ & $8,658.5573$ & -0.0090 \\
\hline $10_{2,9} \leftarrow 9_{2,8}$ & $8,991.0153$ & 0.0224 \\
\hline $10_{4,6} \leftarrow 9_{4,5}$ & $9,129.5526$ & 0.0416 \\
\hline $10_{3,8} \leftarrow 9_{3,7}$ & $9,126.2076$ & -0.0133 \\
\hline $10_{1,9} \leftarrow 9_{1,8}$ & $9,289.055$ & 0.0037 \\
\hline $10_{2,8} \leftarrow 9_{2,7}$ & $9,409.5431$ & -0.0033 \\
\hline $11_{1,11} \leftarrow 10_{1,10}$ & $9,418.9777$ & 0.0028 \\
\hline $11_{0,11} \leftarrow 10_{0,10}$ & $9,479.8798$ & -0.0037 \\
\hline $11_{2,10} \leftarrow 10_{2,9}$ & $9,868.2249$ & 0.0195 \\
\hline $11_{3,9} \leftarrow 10_{3,8}$ & $10,039.2072$ & -0.0217 \\
\hline $11_{1,10} \leftarrow 10_{1,9}$ & $10,164.7117$ & 0.0099 \\
\hline $11_{2,9} \leftarrow 10_{2,8}$ & $10,369.3876$ & -0.0019 \\
\hline $12_{1,12} \leftarrow 11_{1,11}$ & $10,256.3533$ & 0.0049 \\
\hline $12_{0,12} \longleftarrow 11_{0,11}$ & $10,301.6058$ & 0.0014 \\
\hline $12_{2,11} \leftarrow 11_{2,10}$ & $10,739.9261$ & 0.0026 \\
\hline $12_{2,10} \leftarrow 11_{2,9}$ & $11,320.3744$ & -0.0068 \\
\hline $12_{3,10} \longleftarrow 11_{3,9}$ & $10,949.6906$ & -0.0309 \\
\hline $12_{1,11} \leftarrow 11_{1,10}$ & $11,024.3133$ & 0.0072 \\
\hline $13_{1,13} \leftarrow 12_{1,12}$ & $11,091.6847$ & 0.0055 \\
\hline $13_{0,13} \leftarrow 12_{0,12}$ & $11,124.488$ & 0.0023 \\
\hline $13_{2,12} \longleftarrow 12_{2,11}$ & $11,606.0799$ & -0.0285 \\
\hline $13_{2,11} \leftarrow 12_{2,10}$ & $12,260.296$ & 0.0061 \\
\hline $14_{1,14} \leftarrow 13_{1,13}$ & $11,925.3933$ & 0.0044 \\
\hline $14_{1,13} \leftarrow 13_{1,12}$ & $12,699.367$ & -0.0582 \\
\hline $14_{0,14} \longleftarrow 13_{0,13}$ & $11,948.711$ & 0.0015 \\
\hline $15_{1,15} \leftarrow 14_{1,14}$ & $12,757.8488$ & 0.0013 \\
\hline
\end{tabular}

${ }^{a} \Delta \nu=\nu_{\mathrm{obs}}-\nu_{\mathrm{calc}}$. 
Table S.5: The experimental and the theoretical vibrational frequencies (on $\mathrm{HF} / 6-31 \mathrm{G}(\mathrm{d})$ level after scaling) of the TFA and $\mathrm{H}_{2} \mathrm{O}$ monomers. Two scaling factors, one for zero point energy calculations $\left(\rho_{\mathrm{ZPVE}}\right)$ and the other for partition function calculations $\left(\rho_{\mathrm{PF}}\right)$, are derived for both the TFA and the $\mathrm{H}_{2} \mathrm{O}$ monomers.

\begin{tabular}{|c|c|c|c|c|c|}
\hline \multirow{3}{*}{ Mode } & \multicolumn{5}{|c|}{ Frequency $/ \mathrm{cm}^{-1}$} \\
\hline & \multirow[b]{2}{*}{ Experimental } & \multicolumn{4}{|c|}{ Theoretical } \\
\hline & & $\mathrm{PF}^{a}$ & $\Delta \nu / \nu^{b}$ & $\mathrm{ZPVE}^{c}$ & $\Delta \nu / \nu$ \\
\hline \multicolumn{6}{|l|}{ TFA monomer } \\
\hline$\nu_{1}$ & - & 41 & - & 39 & - \\
\hline$\nu_{2}$ & 241 & 240 & $-0.27 \%$ & 230 & $4.50 \%$ \\
\hline$\nu_{3}$ & 247 & 246 & $-0.40 \%$ & 236 & $4.62 \%$ \\
\hline$\nu_{4}$ & 390 & 394 & $0.92 \%$ & 377 & $3.36 \%$ \\
\hline$\nu_{5}$ & 455 & 431 & $-5.20 \%$ & 413 & $9.22 \%$ \\
\hline$\nu_{6}$ & 506 & 507 & $0.25 \%$ & 486 & $4.00 \%$ \\
\hline$\nu_{7}$ & 578 & 585 & $1.26 \%$ & 560 & $3.03 \%$ \\
\hline$\nu_{8}$ & 594 & 595 & $0.20 \%$ & 570 & $4.05 \%$ \\
\hline$\nu_{9}$ & 663 & 675 & $1.83 \%$ & 646 & $2.49 \%$ \\
\hline$\nu_{10}$ & 781 & 811 & $3.88 \%$ & 777 & $0.52 \%$ \\
\hline$\nu_{11}$ & 821 & 814 & $-0.85 \%$ & 779 & $5.06 \%$ \\
\hline$\nu_{12}$ & 1,127 & 1,187 & $5.29 \%$ & 1,136 & $-0.82 \%$ \\
\hline$\nu_{13}$ & 1,184 & 1,264 & $6.73 \%$ & 1,210 & $-2.21 \%$ \\
\hline$\nu_{14}$ & 1,204 & 1,279 & $6.20 \%$ & 1,224 & $-1.70 \%$ \\
\hline$\nu_{15}$ & 1,246 & 1,334 & $7.08 \%$ & 1,278 & $-2.54 \%$ \\
\hline$\nu_{16}$ & 1,417 & 1,479 & $4.35 \%$ & 1,416 & $0.08 \%$ \\
\hline$\nu_{17}$ & 1,827 & 1,933 & $5.82 \%$ & 1,851 & $-1.34 \%$ \\
\hline$\nu_{18}$ & 3,586 & 3,732 & $4.06 \%$ & 3,574 & $0.35 \%$ \\
\hline \multicolumn{6}{|l|}{$\mathrm{H}_{2} \mathrm{O}$ monomer } \\
\hline$\nu_{1}$ & 1,595 & 1,607 & $-0.73 \%$ & 1,633 & $-2.41 \%$ \\
\hline$\nu_{2}$ & 3,652 & 3,582 & $1.90 \%$ & 3,642 & $0.26 \%$ \\
\hline$\nu_{3}$ & 3,756 & 3,687 & $1.83 \%$ & 3,748 & $0.20 \%$ \\
\hline
\end{tabular}

${ }^{a}$ Scaling factor $\rho_{\mathrm{TFA}, \mathrm{PF}}=0.923, \rho_{\mathrm{H}_{2} \mathrm{O}, \mathrm{PF}}=0.880$

${ }^{b} \Delta \nu / \nu=\frac{\nu_{\text {exptl. }}-\nu_{\text {theor. }}}{\nu_{\text {exptl. }}} \times 100 \%$

${ }^{c}$ Scaling factor $\rho_{\text {TFA,ZPVE }}=0.884, \rho_{\mathrm{H}_{2} \mathrm{O}, \mathrm{ZPVE}}=0.895$ 
Table S.6: The predicted vibrational frequencies (on HF/6-31G(d) level after scaling) of the global minimal conformations of TFA- $\left(\mathrm{H}_{2} \mathrm{O}\right)$ and TFA $-\left(\mathrm{H}_{2} \mathrm{O}\right)_{2}$. The scaling factors used for ZPVE and PF calculations were those derived for the TFA monomer in Table S.5.

\begin{tabular}{|c|c|c|c|c|}
\hline \multirow{3}{*}{ Mode } & \multicolumn{4}{|c|}{ Frequency $/ \mathrm{cm}^{-1}$} \\
\hline & \multicolumn{2}{|c|}{ TFA- $\left(\mathrm{H}_{2} \mathrm{O}\right)$} & \multicolumn{2}{|c|}{ TFA- $\left(\mathrm{H}_{2} \mathrm{O}\right)_{2}$} \\
\hline & $\mathrm{PF}^{a}$ & $\mathrm{ZPVE}^{b}$ & $\mathrm{PF}$ & ZPVE \\
\hline$\nu_{1}$ & 38 & 36 & 24 & 23 \\
\hline$\nu_{2}$ & 51 & 49 & 48 & 46 \\
\hline$\nu_{3}$ & 70 & 67 & 61 & 58 \\
\hline$\nu_{4}$ & 149 & 142 & 82 & 79 \\
\hline$\nu_{5}$ & 162 & 155 & 114 & 109 \\
\hline$\nu_{6}$ & 257 & 246 & 170 & 163 \\
\hline$\nu_{7}$ & 262 & 251 & 184 & 176 \\
\hline$\nu_{8}$ & 294 & 281 & 213 & 204 \\
\hline$\nu_{9}$ & 402 & 385 & 231 & 221 \\
\hline$\nu_{10}$ & 408 & 391 & 260 & 249 \\
\hline$\nu_{11}$ & 441 & 422 & 282 & 270 \\
\hline$\nu_{12}$ & 521 & 499 & 313 & 300 \\
\hline$\nu_{13}$ & 600 & 574 & 403 & 386 \\
\hline$\nu_{14}$ & 702 & 673 & 423 & 405 \\
\hline$\nu_{15}$ & 779 & 746 & 444 & 426 \\
\hline$\nu_{16}$ & 826 & 791 & 522 & 500 \\
\hline$\nu_{17}$ & 857 & 821 & 541 & 518 \\
\hline$\nu_{18}$ & 1,214 & 1,163 & 603 & 577 \\
\hline$\nu_{19}$ & 1,275 & 1,221 & 702 & 673 \\
\hline$\nu_{20}$ & 1,314 & 1,258 & 748 & 716 \\
\hline$\nu_{21}$ & 1,372 & 1,314 & 803 & 769 \\
\hline$\nu_{22}$ & 1,513 & 1,448 & 831 & 796 \\
\hline$\nu_{23}$ & 1,598 & 1,625 & 914 & 876 \\
\hline$\nu_{24}$ & 1,907 & 1,826 & 1,224 & 1,172 \\
\hline$\nu_{25}$ & 3,488 & 3,340 & 1,276 & 1,222 \\
\hline$\nu_{26}$ & 3,569 & 3,628 & 1,315 & 1,259 \\
\hline$\nu_{27}$ & 3,676 & 3,737 & 1,381 & 1,323 \\
\hline$\nu_{28}$ & - & - & 1,524 & 1,459 \\
\hline$\nu_{29}$ & - & - & 1,619 & 1,646 \\
\hline$\nu_{30}$ & - & - & 1,635 & 1,662 \\
\hline$\nu_{31}$ & - & - & 1,890 & 1,809 \\
\hline$\nu_{32}$ & - & - & 3,329 & 3,188 \\
\hline$\nu_{33}$ & - & - & 3,448 & 3,506 \\
\hline$\nu_{34}$ & - & - & 3,540 & 3,599 \\
\hline$\nu_{35}$ & - & - & 3,651 & 3,712 \\
\hline$\nu_{36}$ & - & - & 3,662 & 3,723 \\
\hline
\end{tabular}

${ }^{a}$ The scaled vibrational frequencies for calculation of vibrational partition functions PF.

${ }^{b}$ The scaled vibrational frequencies for calculation of zero-point vibrational energies ZPVE 